\title{
Intuitive Interaction in a Mixed Reality System
}

\author{
Shital Desai ${ }^{\mathrm{a}^{*}}$, Alethea Blackler ${ }^{\mathrm{b}}$, Vesna Popovic $^{\mathrm{c}}$ \\ ${ }^{a}$ Queensland University of Technology, Brisbane, Australia \\ ${ }^{b}$ Queensland University of Technology, Brisbane, Australia \\ 'Queensland University of Technology, Brisbane, Australia \\ *Corresponding author e-mail: sh.desai@qut.edu.au
}

\begin{abstract}
Tangible physical systems are more intuitive than Intangible virtual Systems. Mixed reality systems are considered as an alternative to virtual systems, bringing advantages of tangible systems into an interaction. However, past research has mainly focussed on technical aspects of incorporating pervasive-ness and immersive-ness in the virtual systems. This paper reports on an empirical study of intuitive Interaction in a Mixed Reality game system for children and the design aspects that could facilitate intuitive Interaction in such systems. A related samples Friedman's test showed that the Mixed Reality game system demonstrated more intuitive interactions than non-intuitive Interactions. A linear regression analysis further established that the variation in intuitive Interaction in the Mixed Reality system could be statistically significantly explained primarily by physical affordances offered by the Mixed Reality system and to a lesser extent by the perceived affordances in the system. Design guidelines to develop intuitive Mixed Reality systems are discussed. These guidelines should allow designers to exploit the wonders of advances in technology and at the same time allow users to directly interact with the physical real world. This will allow users to access maximal physical affordances, which are primary contributors to intuitive interaction in Tangible and Mixed Reality systems.
\end{abstract}

Keywords: Intuitive Interaction; Mixed Reality Systems; Tangibles; Child Computer Interaction

\section{Introduction}

Developments in hardware technologies have driven impressive technological innovations that let mobile and embedded devices connect to the Web. There is a growing need of people to be able to benefit from these technological innovations and at the same time be able to interact with the real world through tangible systems. 
Children start interacting with tangibles very early in their childhood. Tangibles help children develop their sensory capabilities and put them in control of their learning process, enabling them to learn through personal investigation and exploration (Montessori, 2013). But children are also familiar with modern technology due to increasing everyday use of technology for learning at schools (Walden, 2015) and to play games (Danby et al., 2015). Children are expected to use their past experience and knowledge to play in the virtual space and physical and tangible properties of the real world in the physical space of the Mixed Reality system. Tangibles are more intuitive than the intangible virtual elements (Desai et al., 2015). The coupling between the virtual and physical domains could pose challenges in traversing the boundary between the two domains in Mixed Reality system. The question then arises how designers ensure that mixed reality systems still maintain the intuitiveness of tangible systems.

This research study is an empirical investigation into intuitive interaction in a Mixed Reality game system and design aspects that could facilitate intuitive interaction in a Mixed Reality system.

\section{Background}

A Mixed reality system is a user interface where tangible physical elements and intangible virtual elements co-exist in the same environment. Milgram and Kishino (1994) defined Mixed Reality as being anywhere between the real and virtual environments on the RealVirtual (RV) continuum. The RV continuum can be adapted to discuss Mixed Reality systems in terms of tangibility and physicality of the system and the source of prior knowledge in interaction with the system (Figure 1).

At the extreme left end of the continuum (shown in Figure 1) lie tangible systems representing physical real world associated with interaction using embodied knowledge of the world, also known as sensori-motor knowledge (Blackler et al., 2010; Desai et al., 2015). The intangible systems on the other hand lie at the extreme right end of the continuum representing virtual systems, associated with interaction using experiential knowledge (Desai et al., 2015).

Over the years, designers saw the need to develop systems that allow users to take advantage of the technological innovations but at the same time directly interact with the physical world. The real physical world is either augmented with virtual objects as in Augmented Reality (Azuma et al., 2001) or the virtual world is augmented with real physical objects as in Augmented Virtuality (Regenbrecht et al., 2004). The entire paradigm extending from the tangible to intangible systems is referred to as Mixed Reality Systems. 


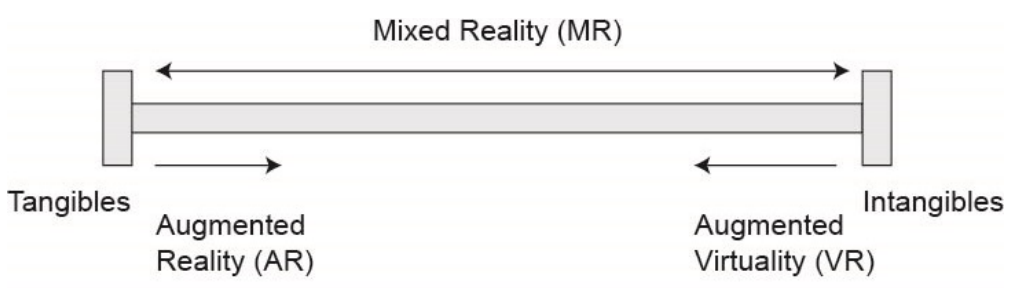

Figure 1 Continuum of Tangible and Intangible Systems (adapted from Milgram and Kishino (1994))

Mixed Reality systems have found their applications in gaming (Crowle et al., 2014), patient rehabilitation (Vogiatzaki et al., 2013), visualization of data (Marks et al., 2014), collaborative coordination in time critical situations (Fischer et al., 2014) and in Education (Gardner and Elliott, 2014). Efforts to develop Mixed Reality systems for children are more prevalent in Education and to some extent in gaming, than in any other field. In one of the first examples of attempts to encourage social interactions in a game, Brederode et al., (2005) developed pOwerball to bring together children with and without learning disabilities to play a game. The game consists of tangible objects that are manipulated on a tabletop to control graphic virtual elements on the tabletop.

Lindgren and Johnson-Glenberg (2013) studied Mixed Reality systems to facilitate embodied and immersive learning in children and developed Meteor, an interactive system that allows children to use their body movements to learn about how objects move in space.

Mixed physical virtual environments in a system could affect the intuitive-ness of the tangible physical environment and thus the Mixed Reality system as a whole. Desai et al. (2015) made an empirical comparison of tangibles and intangibles for intuitive interaction in children and concluded that tangibles are more intuitive than intangibles. They discussed the intuitive interaction in tangibles being the result of children using their sensorimotor knowledge to interact with the tangible system. Intuitive interaction in tangibles is less complex and the encoding and retrieval of associated sensorimotor knowledge is fast. On the other hand, we found that intuitive interaction in Intangibles relies primarily on perceived affordances, derived from prior experience with similar products and features. Intuitive interaction in intangibles is highly complex and the encoding and retrieval of associated experiential knowledge is slow.

Mixed reality systems can pose challenges for children as they are interacting with two interfaces which require knowledge from two different domains. The tendency in the past has been to look at Mixed Reality systems through the lens of pervasiveness (Fischer et al., 2014; Gardner and Elliott, 2014; Ricci et al., 2015). Thus the focus has been more on the technical aspects rather than human centred aspects of design. Attempts have been made to create immersive environments in virtual environments such as in Capture the Flag social gaming environment (Cheok et al., 2006) and Holodeck, a Virtual Reality system for visualisation of scientific data (Marks et al., 2014), instead of maintaining the natural immersive and intuitive capabilities of the physical environment. These systems are mostly based on visual perception using visual systems such as Head Mounted Displays (HMD) and 
Cave Automatic Virtual Environment (CAVE) which could have some impact on the immersive-ness and intuitive-ness of the Mixed Reality systems.

There is no research that looks into intuitive interaction of Mixed Reality systems and aspects that could facilitate intuitive interaction. This is important because in an attempt to allow children to exploit the wonders of technological innovations, designers may end up developing systems that are non-intuitive. This brings with it problems associated with nonintuitive systems such as difficulty in learning which can easily lead to disengagement from the product.

This research study has thus investigated intuitive interaction in a Mixed Reality game system with children. Intuitive interaction in tangible physical systems is a result of children using their sensorimotor knowledge and physical affordances offered by the system and intuitive interaction in Intangibles relies primarily on perceived affordances. This study thus studied physical affordances and perceived affordances as design aspects that could contribute to intuitive interaction in Mixed Reality systems.

\section{Methodology}

An observational study was carried out within People and Systems Lab (PASLab) at Queensland University of Technology (QUT), Brisbane, Australia. Children participated in the study during school holidays. They were asked to bring along a friend or a sibling to play with. The children and their parents were known to the researchers through personal contacts and through their participation in a previous research study.

42 children in the age groups of 5 to 12 years participated in the study. Twenty-one pairs of children were observed playing with a Mixed Reality game system from Tangible Play called Osmo (Figure 2). Osmo allows physical play with a virtual system (IPad). It comes with a reflector and a stand that is attached to an Ipad and four games that can be downloaded as apps from iTunes. The app used for the study is called 'Newton'. Newton works with any objects or drawings that are placed in front of the screen and manipulated to guide falling balls onto targets (Figure 2). The game involves manipulating objects and drawings placed in front of the screen to guide free falling balls onto various targets on the screen. The physical interaction in the game is entirely with the objects in real space and in the context of achieving the goal of directing balls onto the targets. The display screen is used for generating feedback from the manipulation, in relation to the targets on the screen. 


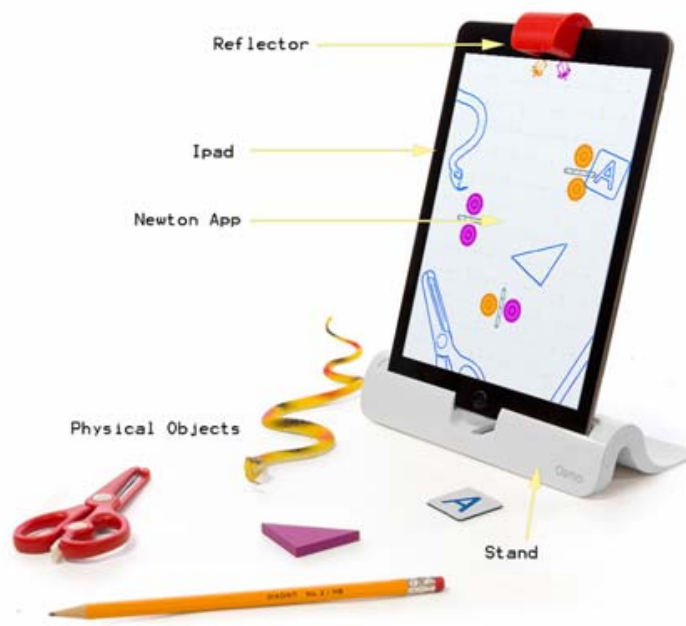

Figure 2 Osmo setup and Newton app.

Children were instructed how to play with the game system and told that they have to work together as a team. The entire game play was video and audio recorded for analysis. Two digital video cameras were used to record the activity (Figure 3). One camera was placed in front of the children and the other on the side to capture the interaction and facial expressions during the playtime.
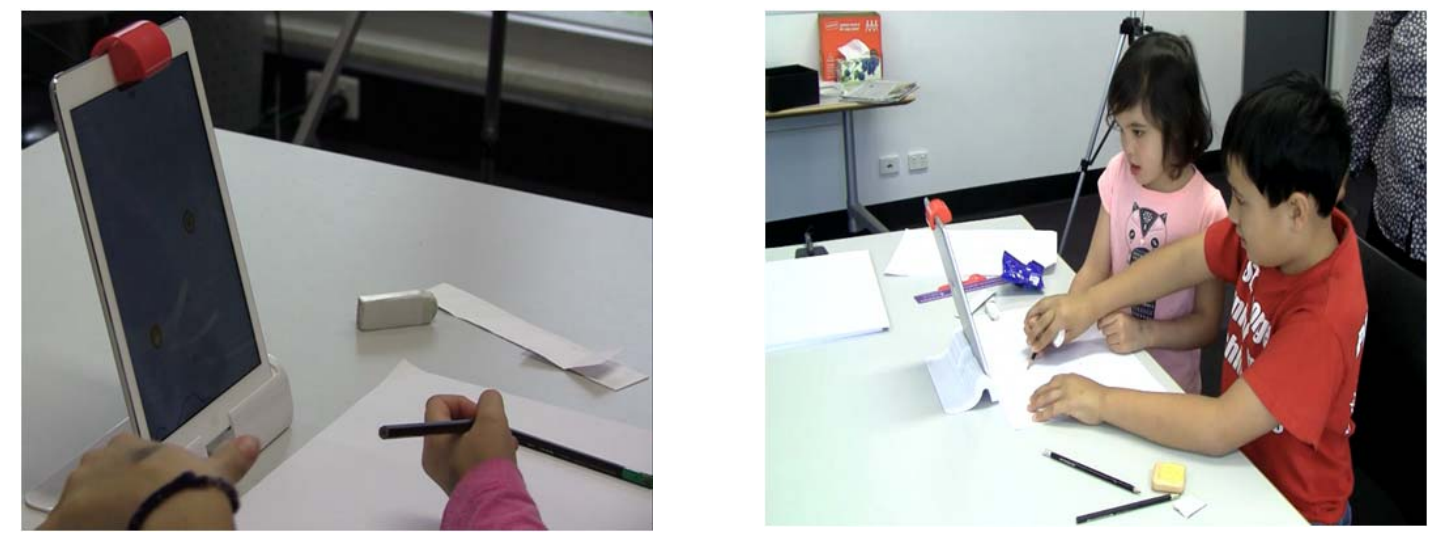

Figure 3 Children playing Osmo. The view of the tablet screen (on the left) and the view of children manipulating objects and drawing in the physical space (on the right)

\section{Analysis}

Audio-video recordings of the game play were coded using Noldus Observer XT 12 to analyse the interaction with the game system. The interaction with the game system was categorised into two main behaviours: Explore and Perform. Explore refers to a behaviour where children were figuring out ways to guide the balls onto the target. They manipulated objects and drawings in front of the tablet, exploring different angles and layouts to guide the balls onto the target. Once they had found the right alignment and layout, they made 
few adjustments to their angular positions to guide the balls onto the target. This behaviour was coded as Perform.

Explore and Perform behaviours were coded for Type of interaction: intuitive, non-intuitive and partially-intuitive and Type of affordance: physical and perceived affordance for each child. Figure 4 shows a part of the coding in Noldus Observer XT 12.

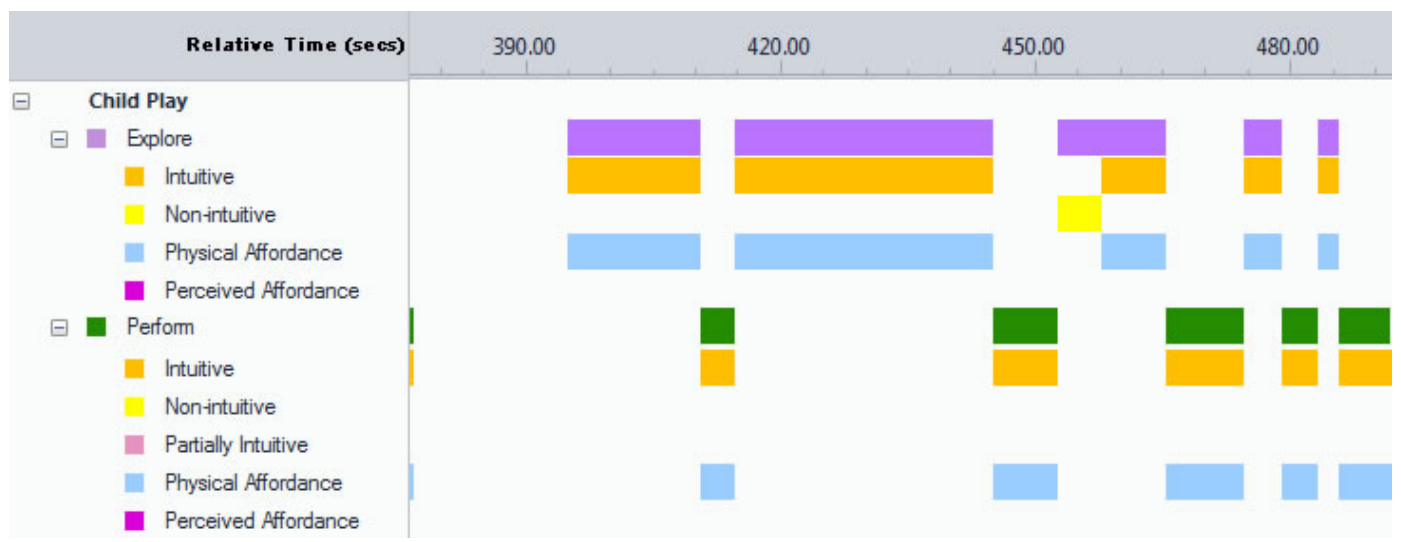

Figure 4 Part of a coding of the game play in Noldus Observer XT 12

\section{Coding Heuristics}

Intuitive interaction involves utilising knowledge gained through other experience(s), is fast (Salk, 1983), and generally non-conscious (Bastick, 2003). The coding heuristics employed to code for intuitive interaction are derivations of methods developed by Blackler et al. (2010).

Non-conscious reasoning - Intuitive interaction does not involve conscious reasoning (Bastick, 2003) but involves actions and decisions which cannot be explained or verbalised (Blackler et al., 2010). Children were considered to be reasoning non-consciously when they could not explain how they guided the balls onto the targets. One of the participants said,

"It is easy, don't you understand this?"

Another participant who chose to draw instead of using objects, said while playing,

"I like to draw, drawing is easy. Do you know I got an award at the assembly for art?"

Although the participant did explain why he chose to draw, the explanation did not match his actions in the game. Such behaviour was also coded as intuitive interaction.

Degree of Certainty - Intuition is associated with high degrees of certainty, confidence and expectation with respect to correct use of a feature (Bastick, 2003; Hammond, 1993;

Woolhouse and Bayne, 2000). When participants were certain and confident about their strategy to guide the balls onto the target, in contrast to trying out various options, the behaviour was coded as intuitive interaction. One participant, while playing the game, said to the other child,

"I know. I know. I got this." 
The above statement not only shows that the participant was certain and confident of her decision but also was reasoning unconsciously because she did not verbalise the actual method that she is going to follow.

Non-intuitive behaviour is associated with conscious reasoning, uncertainty, lack of confidence and unclear expectations with respect to the interaction with the system (ref). While playing one of the levels in the game where four fans have to be spun all at the same time by guiding balls onto the fans, one of the participants said,

"Alright, we need to think about this logically".

Some children did not understand the visual cues in the app on the screen such as a teleporter; they patted their hand on their forehead indicating confusion and said,

"What does this mean?"

When interactions showed signs of intuitive as well as non-intuitive interaction, they were coded as partially-intuitive. For example, a child noticed that the balls were escaping towards the left of the screen instead of being guided towards the target. He picked up a straw and puts it on the left so that balls do not escape anymore. He told the other child,

"Hang on. I have got this! Let's put this [here] so that balls don't run away"

The child clearly verbalised his behaviour (non-intuitive interaction) but was certain and confident about his decision (intuitive interaction).

Physical and perceived affordances were coded as per the following heuristics:

Physical affordance - Objects have spatial and material properties such as colour, texture, composition, size and shape (Hornecker, 2007). The properties of the objects offer a potential use to the user (Maier and Fadel, 2009). People perceive their interaction with these objects by discriminating their properties (Gibson, 1979) and using their sensori-motor knowledge that is derived early in childhood.

Children used spatial orientation of objects and drawings relative to the balls and targets on the screen to decide on the optimum angular position to guide the balls onto the targets. Children aligned pencils and straws at horizontally when the targets were aligned horizontally. When the targets were not aligned horizontally, the objects were aligned at an angle with each other. The properties of the objects do not mean anything in itself, but in relation with other objects in the physical space and elements in the virtual space, children were able to derive appropriate sensori-motor knowledge to align the objects to guide the balls onto the targets (Stoffregen and Mantel, 2015). Hornecker (2007) refers to this relationship as spatial relationship. When children used this spatial relationship to guide the balls onto the targets, the interactions were coded as physical affordance.

Perceived affordance - Perceived affordance is based on past experience and prior knowledge (Blackler et al., 2010; Norman, 2004). People look for clues in the interface to apply their previous knowledge (Dotov et al., 2012). These clues could be incidental/natural, for example weight of a bag, or deliberate, for example a scrollbar in a web explorer. The 
weight of the bag provides a natural clue whether it will be easily carried by the user, without even weighing the bag. In absence of natural clues (physical affordance), children resort to deliberate clues. The scrollbar not only tells users that they can navigate through the web page by sliding the scrollbar but also tells them how much they have read and how much is still left to read. The scrollbar acts like a virtual book mark to the web page. Children perceive such clues using their past experience and knowledge.

The Mixed Reality game system offered familiarity and a set of common metaphors to leverage users' familiarity in interaction. Children used their experience in everyday life and their knowledge about features of tangible objects such as mass, elasticity, rigidity, mobility, etc. One of the children explained his technique of trying to hit the balls with a straw:

"...This is like tennis..."

Some children referred to the simulation of balls hitting a bowl on the screen resulting in a ball emerging from another bowl as a teleporter. One child explained that he had read about it in the book, 'Charlie and the Chocolate Factory'. Interactions where children used their past experience and knowledge to play with the Mixed Reality game system were coded as perceived affordance.

The audio and video data was coded with caution; every observation was checked twice and at times thrice. All coding was done by one researcher and to avoid observer bias, data were coded twice with a break of 15 days in between each coding. Reliability analysis was carried out in Observer XT 12 to determine if there was an agreement between the two sessions of coding carried out by the researcher. Cohen's kappa $(\kappa)$ is a measure of agreement between two sets of coding. Cohen's kappa ( $\mathrm{k}$ ) statistic can range from - 1 to +1 and was found to be 0.92. Based on the guidelines from Altman, (1990), a kappa ( $\mathrm{k}$ ) of 0.92 represents a strong strength of agreement between two sessions of coding. Furthermore, since $p=.00$, kappa (k) coefficient is statistically significantly different from zero.

\section{Results}

The coded data were exported from Observer and then analysed quantitatively using SPSS statistics tool. The objective was to generate reliable generalisable population-based results that are suited to establishing cause-and-effect relationships. Future exploratory and investigative qualitative research will specifically look into these relationships.

\subsection{Intuitive Interaction in Mixed Reality Game System}

The first part of the analysis was to investigate intuitive interaction in the Mixed Reality game System. The boxplot shown in Figure 3 compares the numbers of intuitive, nonintuitive and partially-intuitive interactions in the Mixed Reality system. 


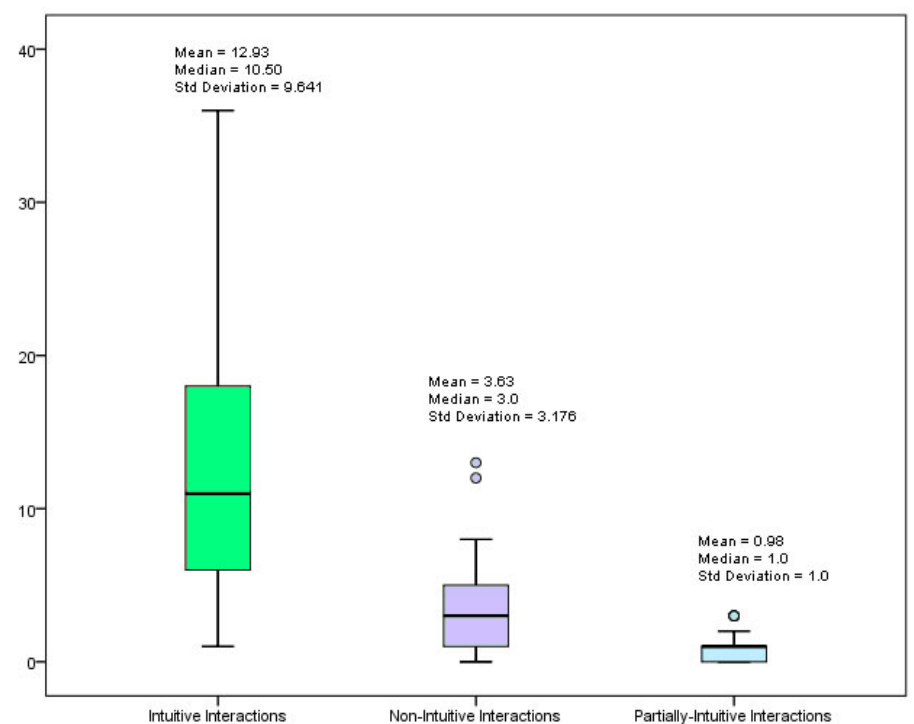

Figure 3 Number of intuitive interactions, non-intuitive interactions and partially-intuitive interactions in a Mixed Reality System

The Mixed Reality game system demonstrated highest number of intuitive interactions followed by non-intuitive interactions. Partially-intuitive interactions were the least demonstrated.

A related Samples Friedman test was run to determine if there were any statistically significant differences between numbers of intuitive, non-intuitive interactions and partiallyintuitive interactions in children playing with the Mixed Reality game system. The Friedman test works by ranking each score of the dependent variables (numbers of intuitive, nonintuitive and partially-intuitive interactions), according to its value, with the smallest rank assigned to the smallest value. The ranks obtained for each of the dependent variables are averaged separately.

The mean ranks obtained for each of the dependent variables and a histogram of rank values of numbers of intuitive interactions, non-intuitive interactions and partially-intuitive interactions is presented in Figure 4. If the shape of the rank distributions is similar, which is the null hypothesis of the Friedman test, the mean rank will be the same for all three types of interactions. However, intuitive interaction has higher mean rank than non-intuitive and partially-intuitive interaction. It is this difference in mean rank that is tested by Friedman test for statistical significance. 


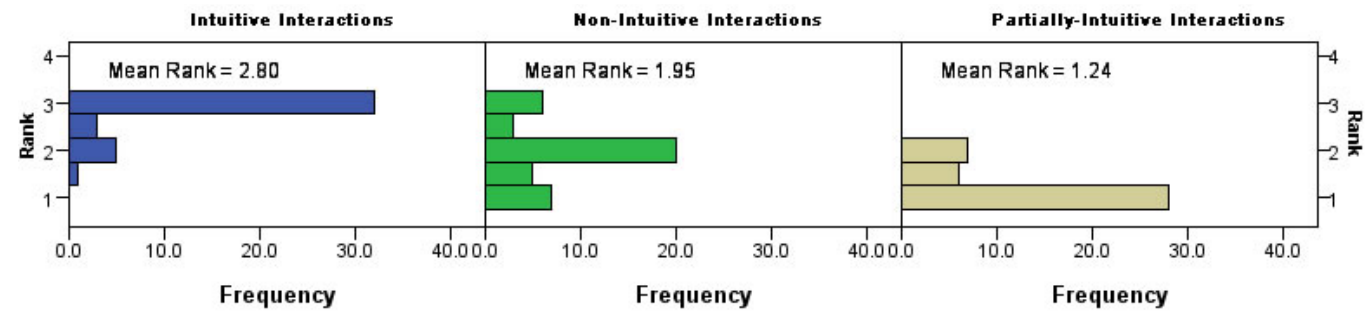

Figure 4 Rank distributions of number of intuitive, non-intuitive and Partially intuitive interactions in Mixed Reality game system.

The rank distributions of numbers of intuitive interactions, non-intuitive interactions and partially-intuitive interactions were not similar, indicating statistically significant difference in the type of interactions, $\chi^{2}(2)=53.006, p<0.05$.

\subsection{Affordances in the Mixed Reality game system}

The intuitive interaction in the Mixed Reality System was further analysed by studying the affordances offered by the game system to facilitate intuitive interaction. A Multiple regression was run to explain how much of the variation in intuitive interaction in the Mixed Reality system can be explained by physical affordances and perceived affordances in the Mixed Reality system. Regression analysis investigates relationships between variables. In contrast to methods such as the Friedman test which indicate if a significant difference exists between the variables, regression analysis determines if physical affordance and perceived affordance influenced intuitive interaction.

Physical and perceived affordances statistically significantly explained $82.5 \%$ of variability in intuitive interaction in the Mixed Reality system, $F(2,39)=95.195, p<0.05$. This suggests that the regression model is a good fit of the data. Comparing the relative contributions of physical and perceived affordance to the intuitive interaction of the system (Figure 5), it was found that physical affordance explained $71.9 \%$ of the variability in the intuitive interaction as compared to Perceived affordance contributing to $28.2 \%$ of the variability.

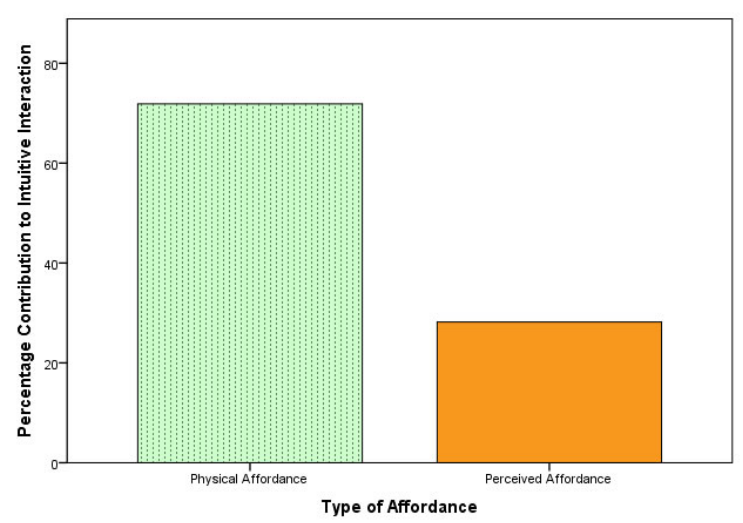

Figure 5 Relative contributions of physical and perceived affordance to the intuitive interaction of the system. 


\section{Discussion}

The results have shown that the Mixed Reality game system demonstrated statistically significantly higher numbers of intuitive interactions as compared to non-intuitive and partially intuitive interactions. Further study into the role of physical affordances and perceived affordances in the intuitive interaction of the Mixed Reality game system also revealed that physical affordances offered by the elements of the game system are the prime contributors of intuitive interaction with the Mixed Reality system.

One of the advantages of direct interaction with objects is that the coupling between cause and effect is quickly observed and its spatial position in space quickly changed if required (Rogers et al., 2002). Children were given an option to either draw or use objects to guide the balls onto the targets on the tablet screen. Some children chose to draw while others preferred to use objects. Some children preferred to draw, but slowly moved on to using objects. This could be because drawing involved erasing number of times before the right alignment was reached. On the other hand, objects required a quick change (cause) in angular position when the balls were missing the target (effect).

Children were able to play intuitively because of the physical affordance offered by the spatial properties of the objects that could be easily manipulated to obtain the correct angular position to achieve the goal of guiding balls onto the target. Children picked long elongated objects such as pencils and straw and drew straight lines to guide the balls onto the targets. Children picked short cardboard strips or an eraser where small deflections were required. While playing levels with multiple targets, children aligned the objects at an angle when the targets were at an angle while they kept the objects horizontal when the targets were placed horizontally.

Perceived affordance also contributed to the intuitive interaction in the Mixed Reality game system. Some children used their past experience and previous knowledge to interpret the meaning of the elements and cues in the app. The spherical virtual objects were intuitively recognised by children as targets. When coloured targets were introduced in the game in one of the levels, children were quick to match the coloured balls onto the same coloured targets. Some children drew tunnels around the target and the gun that released the balls.

It is necessary to look at the contributors of non-intuitive interactions in the game system along with the contributors of the intuitive interaction as it allows designers to come up with design guidelines to develop future intuitive Mixed Reality Systems. Some of the virtual elements in the app either went unnoticed or the children were unable to interpret the meaning of the virtual elements contributing to non-intuitive interactions. Spinning a fan by guiding balls onto it, a teleporter in the form of paired bowls and an accelerator in the form of a flashing arrow could not be reasoned and understood by the children. The meaning of the virtual objects had to be either explained to the children or they consciously tried to reason it out. 
The other cause of non-intuitive interaction was the coupling between the tangible physical and intangible virtual space. They used their previous experience interacting with tablets and virtual systems. Some children started playing the game by swiping on the tablet trying to guide the balls onto the target before realising that the game is controlled through manipulations in the physical space. Children often could not determine the boundaries in the physical space in relation to the virtual space. Some children were unable to understand the disappearance of objects from the virtual space when they moved the objects outside the field of view of the camera, resulting in non-intuitive interactions. Some children used objects in mid-air to strike the balls onto the target, as one child said, "I know this game, I have played this on Xbox". The objects, being too close to the camera, were outside the field of view of the camera and thus these manipulations were not detected in the virtual space. Similar boundary crossings were observed in the horizontal plane, on the left and right of the tablet screen.

However, once Children had figured out the boundary limits of the physical space in relation to the virtual space, they were able to continue playing the game intuitively. One child drew lines on the paper to indicate the boundary limits for moving the objects in the physical space and others were able to able to move the objects back within the limits without prompts. The interpretation of the virtual objects in the virtual space and the coupling between the physical and virtual spaces were the main contributors of non-intuitive interaction in Mixed Reality systems. However, the intuitive interactions in the game system were higher than the non-intuitive interactions, highlighting the importance of physical manipulation of objects in real time and real space in facilitating intuitive interaction in Mixed Reality Systems.

\subsection{Implications in Design}

The mixed reality game system, Osmo consists of a tangible physical space that allows direct interaction and manipulation with physical objects and an intangible virtual space with virtual elements. Intuitive interaction in the Mixed Reality Systems was predominantly due to physical affordances offered by the spatial orientation of the physical objects in the physical space in relation to the virtual elements in the virtual space.

non-intuitive interaction in the mixed reality game system is predominantly due to the virtual elements, the meanings of which were not understood by the children and the challenges posed by the coupling between the physical and virtual spaces. Children spent some time learning about the virtual elements and the boundary issues before they started playing intuitively.

Although the Mixed Reality game system demonstrated non-intuitive interactions due to above mentioned issues, there were higher numbers of intuitive interactions than nonintuitive interactions in the game system primarily due to the physical affordances offered by the direct interaction and manipulation of the objects in the physical space. This is in line with Blackler's (2008) continuum of intuitive interaction, according to which the simplest 
form of intuitive interaction is through physical affordance which is derived from sensorimotor knowledge. Encoding and retrieval of sensorimotor knowledge is fast and it is acquired very early in childhood. Population stereotypes (such as clockwise to increase or red for stop) is the second most accessible form of intuitive use followed by perceived affordances. The higher end of the continuum contributes to high complexity in design for intuitive use (Blackler, 2008) and requires maximum encoding and retrieval time.

Desai et al. (2015) compared intuitive interaction in a tangible physical system to that in an equivalent intangible virtual system using continuum of intuitive interaction. They found that intuitive interaction in physical systems is derived from sensorimotor knowledge, physical affordances offered by spatial and material features naturally inherent in the tangibles and from experiential knowledge acquired from prior experience with the physical properties of similar and other physical systems. Intuitive interaction in virtual systems relies heavily on perceived affordances, derived from prior experience with similar products and features. Intuitive interaction in virtual systems is also governed by population stereotypes associated with the technology such as tablets and touch screens are associated with swiping left, right, up and down on the screen.

Based on the discussion above on factors contributing to intuitive and non-intuitive interaction in the Mixed-Reality game system, the following design guidelines could help to insure intuitive interaction in Mixed Reality Systems:

1. Use of physical affordances - Intuitive interaction in tangible and Mixed Reality systems is primarily due to physical affordances. Facilitating user inputs to the system through direct interaction and manipulation of physical elements in the physical space allows users to take advantage of the physical affordances offered by the elements in the system. The virtual system provides feedback on the manipulation and interaction in the physical space.

2. Use of perceived affordances - The virtual elements in the virtual space should be designed keeping the past experience and prior knowledge of the users in mind. The act of spinning a virtual fan is not known to children, so cues in the form of a simulation of balls falling on a fan resulting in its spin could speed up the learning process.

3. Dimensions of interaction - If the virtual space is in two-dimensions, such as the tablet screen in Osmo, limiting the interactions and manipulations in the physical space in two-dimensions, such as moving objects in the horizontal plane, help users to traverse the coupling between physical and virtual space.

4. Size of interaction spaces - The size of the physical space and the virtual space in a Mixed Reality System are usually not the same. This could result in physical manipulations and interactions in the physical space going out of the limits of the virtual space. The boundaries in the physical space in relation to the virtual space should be specified. For example, simple lines in the physical space outlining the boundaries in the field of view of the camera could reduce the time taken to learn 
about the boundaries. Alternatively, a feedback from the system to move back within the boundary limits could help train people to determine the boundaries.

These guidelines are some ways in which Mixed Reality systems could be designed for intuitive interaction and probably also for immersive interaction.

\section{Conclusions and Future Research}

Prior research has shown that tangibles are more intuitive than intangible systems. Mixed reality systems incorporate both tangibles and intangibles in one system. A question then arises on the intuitiveness of Mixed Reality Systems. This study has carried out an empirical investigation on intuitive interaction in a Mixed Reality game system and the aspects that facilitate intuitive interaction.

Intuitive interaction in Mixed Reality systems is primarily derived from sensorimotor knowledge and physical affordances offered by the tangibles and from prior experience with the physical properties of similar and other tangibles. The coupling between the physical and virtual space and children unable to reason with the virtual elements in the virtual space were identified as contributors to non-intuitive interaction in the system.

Some design guidelines to develop intuitive Mixed Reality systems were discussed. Further research into different configurations of Mixed Reality systems, such as body interactions and gesture-based interactions, is required to further build on the guidelines. This study is important as it investigates Mixed Reality systems from the perspective of a human centred design approach rather than from the perspective of looking at technical aspects of incorporating immersive-ness and pervasive-ness in a virtual space.

Acknowledgements: We thank all parents and children for their support and participation in this research study.

\subsection{References}

Azuma, R., Baillot, Y., Behringer, R., Feiner, S., Julier, S., Maclntyre, B., 2001. Recent advances in augmented reality. IEEE Comput. Graph. Appl. 21, 34-47. doi:10.1109/38.963459

Bastick, T., 2003. Intuition: Evaluating the construct and its impact on creative thinking. Stoneman \& Lang.

Blackler, A., 2008. Intuitive interaction with complex artefacts : empirically-based research. VDM Verlag, Saarbrücken, Germany.

Blackler, A., Popovic, V., Mahar, D., 2010. Investigating users' intuitive interaction with complex artefacts. Appl. Ergon. 41, 72-92. doi:10.1016/j.apergo.2009.04.010

Brederode, B., Markopoulos, P., Gielen, M., Vermeeren, A., de Ridder, H., 2005. pOwerball: the design of a novel mixed-reality game for children with mixed abilities. Interact. Des. Child. 32-39. doi:10.1145/1109540.1109545

Cheok, A.D., Sreekumar, A., Lei, C., Thang, L.N., 2006. Capture the flag: Mixed-reality social gaming with smart phones. IEEE Pervasive Comput. 5, 62-69. doi:10.1109/MPRV.2006.25 
Crowle, S., Boniface, M., Poussard, B., Asteriadis, S., 2014. A design and evaluation framework for a tele-immersive mixed reality platform. Lect. Notes Comput. Sci. (including Subser. Lect. Notes Artif. Intell. Lect. Notes Bioinformatics) 8853, 151-158. doi:10.1007/978-3-319-13969-2_12

Danby, S., Davidson, C., Theobald, M., Houen, S., Thorpe, K., 2015. Playing with Technology: Young Children Making Sense of Technology as Part of Their Everyday Social Worlds. Multidiscip. Perspect. Play From Birth to Beyond.

Desai, S., Blackler, A., Popovic, V., 2015. Intuitive use of tangibles, in: IASDR.

Dotov, D.G., Nie, L., De Wit, M.M., 2012. Understanding affordances: history and contemporary. choices 33, 269-298.

Fischer, J.E., Jiang, W., Kerne, A., Greenhalgh, C., Ramchurn, S.D., Reece, S., Pantidi, N., Rodden, T., 2014. Supporting Team Coordination on the Ground: Requirements from a Mixed Reality Game. COOP 2014 - Proc. 11th Int. Conf. Des. Coop. Syst. 49-67. doi:10.1007/978-3-319-06498-7_4

Gardner, M., Elliott, J.B., 2014. The Immersive Education Laboratory : understanding affordances, structuring experiences, and creating constructivist, collaborative processes, in mixed-reality smart environments. Trans. Futur. Intell. Educ. Environ. 1, 1-13.

Gibson, J.J., 1979. The Theory of Affordances in the Ecological Approach to Visual Perceptual.

Hammond, K.R., 1993. Naturalistic decision making from a Brunswikian viewpoint: Its past, present, future. Decis. Mak. action Model. methods 205-227.

Hornecker, E., 2007. Physical affordances considered harmful!?, in: Second International Workshop on Physicality. p. 15.

Lindgren, R., Johnson-Glenberg, M., 2013. Emboldened by Embodiment Six Precepts for Research on Embodied Learning and Mixed Reality. Educ. Res. 42, 445-452. doi:10.31020013189X13511661

Maier, J.R.A., Fadel, G.M., 2009. Affordance based design: a relational theory for design. Res. Eng. Des. 20, 13-27.

Marks, S., Estevez, J.E., Connor, A.M., 2014. Towards the Holodeck: Fully Immersive Virtual Reality Visualisation of Scientific and Engineering Data. Proc. 29th Int. Conf. Image Vis. Comput. New Zeal. - IVCNZ'14 42-47. doi:10.1145/2683405.2683424

Milgram, P., Kishino, F., 1994. A taxonomy of mixed reality visual displays. IEICE Trans. Inf. Syst. 77, 1321-1329.

Montessori, M., 2013. The montessori method. Transaction Publishers.

Norman, D.A., 2004. Emotional design: Why we love (or hate) everyday things. New York.

Regenbrecht, H., Lum, T., Kohler, P., Ott, C., Wagner, M., Wilke, W., Mueller, E., 2004. Using Augmented Virtuality for Remote Collaboration. Presence Teleoperators Virtual Environ. 13, 338354. doi:10.1162/1054746041422334

Ricci, A., Piunti, M., Tummolini, L., Castelfranchi, C., 2015. The mirror world: Preparing for mixedreality living. IEEE Pervasive Comput. 14, 60-63. doi:10.1109/MPRV.2015.44

Rogers, Y., Scaife, M., Gabrielli, S., Smith, H., Harris, E., 2002. A Conceptual Framework for Mixed Reality Environments: Designing Novel Learning Activities for Young Children. Presence Teleoperators Virtual Environ. 11, 677-686. doi:10.1162/105474602321050776

Salk, J., 1983. Anatomy of reality. Columbia University Press New York.

Stoffregen, T.A., Mantel, B., 2015. Exploratory movement and affordances in design. Artif. Intell. Eng. Des. Anal. Manuf. 29, 257-265. doi:10.1017/S0890060415000190

Vogiatzaki, E., Gravezas, Y., Solutions, I.S.A.T., 2013. Rehabilitation System for Stroke Patients using Mixed-Reality and Immersive User Interfaces.

Walden, R., 2015. Schools for the Future. Springer. 
Woolhouse, L.S., Bayne, R., 2000. Personality and the use of intuition: individual differences in strategy and performance on an implicit learning task. Eur. J. Pers. 14, 157-169. doi:10.1002/(SICI)1099-0984(200003/04)14:2<157::AID-PER366>3.0.CO;2-L

About the Authors:

Shital Desai (M.S) is a PhD student Discipline in Industrial Design at QUT, Brisbane, Australia. She is currently working on her thesis which looks at developing a framework for designing products that facilitate Embodied Intuitive Interaction in children. She is a recipient of research bursary from Design Research Society (UK). (sh.desai@qut.edu.au)

Alethea Blackler (PhD) is an Associate Professor and Head of Discipline in Industrial Design at QUT, Brisbane, Australia. Her principle area of research interest is intuitive interaction, in which she is one of the world leaders. She pioneered the first empirical work in this field. (a.blackler@qut.edu.au)

Vesna Popovic (PhD) is a Professor in Industrial Design at Queensland University of Technology, Brisbane, Australia. Her research focus is within experience, expertise and intuitive interaction. Vesna is a Fellow of the Design Research Society (UK) and Design Institute of Australia. (v.popovic@qut.edu.au). 